\title{
Desarrollo de un software para el modelamiento de precipitación de asfaltenos y parafinas incorporando daño de formación por depositación de asfaltenos en yacimientos de petróleo
}

\author{
Development of software for asphaltene and wax precipitation \\ modeling incorporating formation damage by asphaltene \\ deposition in oil reservoirs
}

\author{
Jairo Antonio Sepúlveda, Israel Yuseth Patío \\ y Manuel Fernando Gutiérrez
}

\section{Resumen}

En este articulo se describen diferentes modelamientos termodinámicos para predecir la precipitación de material orgánico (parafinas y asfaltenos) utilizando ecuaciones de estado que permiten predecir el momento en el cual iniciará la precipitación. Se describe un modelo físico-mecánico para la depositación de asfaltenos que cuantifica la cantidad de asfalteno depositado alrededor de un pozo a diferentes intervalos de tiempo. Posteriormente se desarrolla un programa denominado SMAP (Software para el Modelamiento de Asfaltenos y Parafinas) que permite caracterizar y fraccionar el componente $(\mathrm{Cn}+)$, agrupar pseudocomponentes, modelar la precipitación de asfaltenos y parafinas, cuantificar, analizar y predecir el daño de formación por depositación de asfalteno en el yacimiento. Al comparar los resultados con datos experimentales encontrados en otros articulos, con el CMG y con algunos datos de campos reales, es posible hacer la validación y una evaluación precisa del proyecto.

Palabras clave: Comportamiento de fases; precipitación de parafinas; precipitación de asfaltenos; depositación de asfaltenos; modelos termodinámicos; ecuación de estado; daño de formación por depositación de sólidos.

\begin{abstract}
This article describes different thermodynamic models to predict the precipitation of organic material (paraffins and asphaltenes) using state equations that predict the time at which precipitation starts. We describe a physicomechanic model for asphaltene deposition that quantifies the amount of asphaltene deposited around a well at different time intervals. Was subsequently developed a program called SMAP that allows characterize and splitting the $\mathrm{Cn}+$ component, lumping the pseudocomponents, model the asphaltene and paraffin precipitation. quantify, analyze and predict formation damage by asphaltene deposition in the reservoir. By comparing the results with experimental data found in other articles, the CMG and some actual field data, it is possible to do the validation and accurate assessment of the project.
\end{abstract}

Keywords: Phase behavior; Paraffin precipitation; Asphaltene precipitation; Asphaltene deposition: Thermodynamic models; Equation of state; Formation damage by Asphaltene deposition.

\footnotetext{
I MSc. en Ingenieria de Petróleos, Universidad Surcolombiana, Neiva-Huila jsepulveda usco.edu.co

2 Ingeniera de Petróleos, Universidad Surcolombiana, Neiva-Huila ipvusethra gmail.com

3 Ingeniero de Petróleos, Universidad Surcolombiana, Neiva-Huila mac_manael1990@hotmail.com
} 


\section{Introducción}

La depositación de material orgánico en los procesos de producción de crudo es uno de los problemas más comunes en la industria petrolera. Variaciones en la presión y fuertes caídas en la temperatura ocasiona que las parafinas y el asfalteno se precipiten fuera del fluido que los transporta ocasionando taponamiento, disminuyendo así el área disponible al flujo, lo que genera una disminución en la rata de producción de hidrocarburo.

Debido a los costos operacionales que representa este tipo de problemas en la industria, muchos autores se han dado a la tarea de estudiar y comprender el comportamiento de las parafinas y asfaltenos y asi poder generar modelos que permitan realizar una predicción de la precipitación de los mismos. Un modelo que realice una acertada predicción es indispensable pues permite que las operaciones se realicen con el fin de evitar las condiciones propicias que generen precipitación.

Los objetivos de esta investigación es hacer una recopilación de los modelos de parafinas y asfaltenos, posteriormente se procede a seleccionar los mejores modelos y desarrollarlos mediante un programa computacional. Este software debe permitir al usuario conocer las condiciones de precipitación de sólidos para la toma de decisiones. Los modelos seleccionados para parafinas segín su fase solida la podemos clasificar en un Modelo de solución sólida (Won, 1985; Won 1989) y un Modelo de fase multisólida (multisólido (LiraGaleana et al, 1996; Nichita et al, 2001). En la literatura hay muchos modelos referentes a precipitación de asfaltenos de acuerdo a diversas teorias planteadas sobre su estabilidad y precipitación; Modelo de termodinámico (Leontaritis, 1987), Modelo de solución polimérica (Hirschberg et al., 1988), Modelo de sólidos (Gghiem el al., 1993), Modelo de solución regular (Chung, 1992), Modelo de PC-SAFT (Chapman et al, 1990). Modelo de micelización (Victorov et al, 1996).

La precipitación de asfaltenos en el espacio poroso de la roca del yacimiento puede propiciar una depositación que al taponar la garganta del poro, altera las propiedades petrofisicas de la roca disminuyendo la porosidad y la permeabilidad, aumentando progresivamente el factor de daño cerca a la cara del pozo afectando seriamente el aporte de fluido del yacimiento al pozo. Contar con un programa computacional que simule la depositación es indispensable para predecir el momento de aparición de solidos orgánicos en campos con estos problemas, para esto es necesario desarrollar un modelo fisico-químico mediante la combinación de un modelo de precipitación (Nghiem et al, 1993) y un modelo de depositación (Leontaritis, 1996) logrando de esta manera un modelo que ayuda a anticipar el problema permitiendo acciones preventivas óptimas que reduzcan en lo posible las pérdidas económicas de procesos de control y reparación.

\section{Metodología}

\subsection{Modelamiento de parafinas}

Muchos son los autores que han investigado el comportamiento de las parafinas concluyendo que su cristalización está directamente relacionada con una disminución de temperatura; en base a ésto y la gran variedad de inconvenientes que se presentan debido a la precipitación y depositación de éstas, se hace necesario tener a la mano modelos que permitan una predicción eficiente de la temperatura de aparición de cera (WAP).

La mayor diferencia entre modelos está en la descripción de la no idealidad de las fases; así para sistemas a baja presión la fase liquida se puede considerar ideal mientras que para presiones altas, la fase líquida es generalmente descrita usando una ecuación de estado. A la fase sólida se le han dado dos enfoques, obteniendo asi, dos modelos diferentes:

\subsubsection{Modelo de solución sólida}

Se asume que la precipitación es una solución sólida ideal (coeficiente de actividad es igual a 1; no ocurre cambio en el volumen del soluto y del solvente cuando sucede la mezcla, sino que los volúmenes son aditivos) 
dentro de un numero de distintas fases cristalinas, las fugacidades en la fase vapor y líquida pueden obtenerse de una ecuación de estado.

Debido al supuesto de soluciones ideales (Won, 1985; Won 1989), se asume la relación de coeficientes de actividad igual a la unidad. En este trabajo, Ecuación 1, para determinación de la constante inicial de equilibrio es entonces:

$$
k_{i}^{S L}=e^{\left(\frac{\mathrm{Hf}_{i}}{\mathrm{RI}^{*}}\left(1-\frac{\mathrm{T}}{\mathrm{Tf}_{i}}\right)\right)}
$$

Donde la temperatura (Ecuación 2) y entalpia de fusión (Ecuación 3) son determinadas mediante las siguientes correlaciones:

$$
\begin{gathered}
\mathrm{Tf}_{\mathrm{i}}=374.5+0.02617 \mathrm{Mw}_{\mathrm{i}}-20172 / \mathrm{Mw}_{\mathrm{i}}(2) \\
\mathrm{Hf}_{\mathrm{i}}=0.9 \mathrm{Mw}_{\mathrm{i}}^{0.55 * \mathrm{Tf}_{\mathrm{i}}(3)}
\end{gathered}
$$

Mediante la correlación empirica presentada por Chung (Chung, 1992), se halla el volumen molar (Ecuación 4) y con éste la fracción en volumen del componente $i$ en cada una de las fases (Ecuaciones 5,6 y 7):

$$
\begin{gathered}
\mathrm{Vm}=\sum 3.8^{*} \mathrm{Mw}_{\mathrm{i}}^{0.786}(4) \\
\mathrm{x}_{\mathrm{i}}=\sum \frac{\mathrm{x}_{\mathrm{i}}^{*} \mathrm{Vm}}{\sum \mathrm{X}_{\mathrm{i}}^{*} \mathrm{Vm}}(5) \\
\mathrm{s}_{\mathrm{i}}=\sum \frac{\mathrm{S}_{\mathrm{i}}^{*} \mathrm{Vm}}{\sum \mathrm{S}_{\mathrm{i}}{ }^{*} \mathrm{Vm}}(6) \\
\mathrm{y}_{\mathrm{i}}=\sum \frac{\mathrm{Y}_{\mathrm{i}} * \mathrm{Vm}}{\sum \mathrm{Y}_{\mathrm{i}}{ }^{*} \mathrm{Vm}}(7)
\end{gathered}
$$

Con las Ecuaciones 2 y 3 , se determinan temperatura y entalpia para asi poder determinar el parámetro de solubilidad de cada fase como se observa en le Ecuación 8:

$$
\delta_{1}^{f}=7.02+1.6^{*}\left(1-e^{\frac{4.649422\left(\mathrm{Mw}_{1}-58.124^{0.79151}\right)}{1441.376}}\right)(8)
$$
9:

A continuación se hallan unos parámetros de solubilidad promedio de cada fase como sigue en la Ecuación

$$
\delta_{\mathrm{f}}^{\mathrm{prom}}=\sum \delta^{\mathrm{f}} \mathrm{x}(9)
$$

Con los parámetros de solubilidad promedios de cada fase se puede ahora determinar los coeficientes de actividad de las fases líquida y sólida como se muestra en la Ecuación 10:

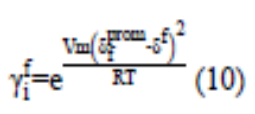

Remplazando estos valores en la Ecuación 11, se puede determinar la constante de equilibrio: 


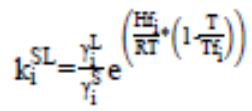

Se determina la composición molar de las fases mediante la ecuación de Rachford Rice (Ecuación 12), usando el método iterativo de Newton Rahpson (Nghiem., 1984). Primero se hallan las moles de la fase sólida y con ésta se determinan las fracciones del componente $i$ en cada fase (Ecuaciones 13 y 14):

$$
\begin{gathered}
f\left(\mathrm{n}_{\mathrm{s}}\right)=\sum_{\mathrm{i}=1}^{\mathrm{n}} \frac{\mathrm{z}_{\mathrm{i}}\left(\mathrm{K}_{\mathrm{i}}^{\mathrm{SL}}-1\right)}{1+\mathrm{n}_{\mathrm{i}}\left(\mathrm{K}_{\mathrm{i}}^{\mathrm{SL}}-1\right)}=0(12) \\
\mathrm{s}_{\mathrm{i}}=\frac{\mathrm{z}_{\mathrm{i}} \mathrm{K}_{\mathrm{i}}^{\mathrm{SL}}}{1+\mathrm{n}_{\mathrm{S}}\left(\mathrm{K}_{\mathrm{i}}-1\right)}(13) \\
\mathrm{x}_{\mathrm{i}}=\frac{\mathrm{z}_{\mathrm{i}}}{1+\mathrm{n}_{\mathrm{S}}\left(\mathrm{K}_{\mathrm{i}}^{\mathrm{SL}}-1\right)}(14)
\end{gathered}
$$

Luego, como se observó en el capitulo anterior se determinan el coeficiente de fugacidad y la fugacidad del sólido y del liquido. Para el cálculo WAP (determinación de la temperatura a la que precipita el liquido), el programa, a una presión constante empieza a variar temperatura y calcula, mediante el procedimiento descrito arriba, las moles de sólido hasta obtener la primera partícula precipitada. Se determinan luego algunas propiedades de las fases (peso molecular, densidad, volumen corregido, densidad corregida).

\subsubsection{Modelo de fase multisólida}

En el modelo multisólido, la fase sólida (cera) se compone de varios componentes puros. Los estudios muestran dos modelos principales para aplicar el concepto de modelo multisólido (Lira-Galeana et al, 1996; Nichita et al, 2001). Los otros modelos multisólidos son similares.

Para modelar el equilibrio entre el aceite y la parafina se requiere una relación termodinámica precisa. Existe una relación de equilibrio entre la fugacidad de la fase líquida (aceite) y la fugacidad de la fase sólida (parafina) como se muestra en la Ecuación 15:

$$
\ln \frac{f_{1}}{f_{s}}=\frac{H f_{i}}{R^{*} T f_{i}}\left[\frac{T f_{i}}{T}-1\right]+\frac{H f_{t, i}}{R^{*} T_{t, i}}\left[\frac{T_{t, i}}{T}-1\right]-\frac{C_{p i j}}{R}\left[\ln \frac{T}{T_{i}}+\frac{T f_{i}}{T}-1\right](15)
$$

En el modelo multisólido, el número de componentes precipitado debe ser obtenido por condiciones de análisis de estabilidad como se observa en la Ecuación 16.

$$
f_{i}^{Z}(P, T, Z i)-f_{i}^{S, p u r o}(P, T)>0 i=1, \ldots n(16)
$$

Donde $f_{i}^{z}(P, T, Z i)$, es la fugacidad del componente en la mezcla a presión $\mathrm{P}$ y temperatura definidas, donde $\mathrm{S}$ hace referencia a la fase sólida. La fugacidad del componente en la mezcla puede ser calculada como sigue en las Ecuaciones $17 \mathrm{y} 18$ :

$$
\mathrm{f}_{\mathrm{i}}^{\mathrm{Z}}(\mathrm{P}, \mathrm{T}, \mathrm{Zi})=\mathrm{f}_{\mathrm{i}}^{\mathrm{L}, \text { puro }}(\mathrm{P}, \mathrm{T}) * \gamma_{\mathrm{i}}^{\mathrm{L}} * \mathrm{X}_{\mathrm{i}}^{\mathrm{L}}(17)
$$

Asi:

$$
\mathrm{f}_{i}^{\mathrm{L}, \mathrm{puro}}(\mathrm{P}, \mathrm{T}) * \gamma_{\mathrm{i}}^{\mathrm{L}} * \mathrm{X}_{\mathrm{i}}^{\mathrm{L}}-\mathrm{f}_{\mathrm{i}}^{\mathrm{Z}}(\mathrm{P}, \mathrm{T}, \mathrm{Zi})(18)
$$


El coeficiente de actividad $\gamma_{i}^{L}$, es hallado usando modelos descritos en el modelo anterior por ejemplo el modelo de solución regular. La solución ideal se enfoca en considerar $\gamma_{i}^{L}=1$. La relación de fugacidad puede ser calculada como se muestra en la ecuación 15. La temperatura de fusión $T_{f}$ de alcanos normales (Ecuación 19), se estima de la siguiente correlación propuesta por Won (Won., 1986).

$$
\mathrm{T}_{\mathrm{fi}}=374.5+0.2617 * \mathrm{Mw}_{\mathrm{i}}-\frac{20172}{\mathrm{Mw}_{\mathrm{i}}}
$$

Para la estimación de la temperatura de transición de estado sólido, $T_{t r, i}$, se utiliza la correlación observada en el Ecuación 20, propuesta por Nichita (Nichita et al, 2001):

$$
\mathrm{T}_{\mathrm{tr}, \mathrm{i}}=366.39775+0.03609 * \mathrm{Mw}_{\mathrm{i}}-\frac{20879}{\mathrm{Mw}_{\mathrm{i}}}(20)
$$

En la Ecuaciones 19 y 20, la temperatura está expresada en $\mathrm{K}$, y el $M w$ es el peso molecular del componente. Para el cálculo de la fusión y la entalpia de transición sólido-sólido de los alcanos normales (Nichita et al, 2001) sugieren las siguientes correlaciones (Ecuaciones 21 y 22$)$, para Mw $>282\left(\frac{\mathrm{g}}{\mathrm{gmol}}\right)$ :

$$
\begin{gathered}
\Delta \mathrm{Hf}_{\mathrm{i}}=0.1186^{*} \mathrm{Mw}_{\mathrm{i}} * \mathrm{~T}_{\mathrm{fi}}(21) \\
\Delta \mathrm{H}_{\mathrm{i}, \mathrm{tr}}=0.0577^{*} \mathrm{Mw}_{\mathrm{i}} * \mathrm{~T}_{\text {tr,i }}(22)
\end{gathered}
$$

$\mathrm{Y}$ para Mw $<282\left(\frac{\mathrm{g}}{\mathrm{g} m 01}\right)$ se expresa la entalpia total de fusión por la siguiente correlación:

$$
\Delta \mathrm{Hf}_{\mathrm{i}}^{\mathrm{t}}=0.1777 * \mathrm{Mw}_{\mathrm{i}} * \mathrm{~T}_{\mathrm{fi}}(23)
$$

En las Ecuaciones 21 a 23, $\Delta H$ está expresada en cal/mol. Para cálculos de la capacidad calorífica entre la fase sólida y la liquida, $\Delta C_{p i}$, la siguiente correlación (Ecuación 24) es propuesta por Pedersen (Pedersen et al., 1991):

$$
\Delta \mathrm{C}_{\mathrm{pi}}=0.3033 * \mathrm{Mw}_{\mathrm{i}}-4.635 \times 10^{-4} * \mathrm{Mw}_{\mathrm{i}} * \mathrm{~T}(24)
$$

Donde, $\Delta C_{p i}$ se expresa en cal $/ \mathrm{mol} \mathrm{K}$ y $\mathrm{T}$ en $\mathrm{K}$. Finalmente para componentes precipitantes, el equilibrio termodinámico puede ser escrito como sigue en la Ecuación 25:

$$
f_{i}^{L}\left(P, T, x_{i}^{L}\right)=f_{i}^{S, ~ p u r o ~}(P, T) i=1 \ldots . . . n_{s}(25)
$$

Donde, $n_{s}$ es el número de componentes precipitantes. Usando la correlación de análisis de estabilidad y el balance de materia para componentes precipitantes y no-precipitantes, la fracción molar y la composición de la fase Sólida pueden ser obtenidas.

\subsection{Modelamiento de asfáltenos}

Se ha demostrado que los cambios de temperatura, presión y composición del crudo causa la precipitación de estos asfáltenos. La variación de alguno de estos parámetros ocasionara la desestabilización del sistema crudo-asfálteno y por lo tanto se genera la floculación de asfálteno y la formación de material insoluble en el crudo líquido. La precipitación por encima de la presión de saturación, alcanza su valor máximo alrededor de la presión de saturación y disminuye a medida que la presión disminuye más. Estas precipitaciones pueden causar serios problemas ya que al depositarse, estos sólidos en las gargantas de los poros pueden taponar la formación y/o tuberias de facilidades de producción. 
Algunos factores que causan precipitación de asfálteno fueron mencionados anteriormente, no obstante existen teorías y modelos basados en distintas teorias microscópicas que tratan de explicar este fenómeno y que a la vez sirven para predecir los distintos mecanismos de precipitación de asfältenos. En esta investigación se ha realizado una exhaustiva clasificación de modelos existentes en la actualidad, como conclusión hemos clasificado 6 tipos de modelos los cuales tienen como objetivo modelar la precipitación de asfáltenos cuando las condiciones iniciales cambian.

\subsubsection{Modelo termodinámico coloidal}

De acuerdo con este modelo, los asfaltenos sólo pueden permanecer en una fase líquida homogénea debido a que forman micelas protegidos por una capa exterior de resinas. La precipitación de asfaltenos está determinada por la relación entre el potencial químico, $\mu$, de las resinas en la fase líquida y el potencial químico de resinas en el micelas de asfalteno (Ecuación 26).

$$
\mu_{\text {resina }}^{\text {Micela de asfalteno }}=\mu_{\text {resina }}^{\text {Fase liquida }}
$$

E1 modelo termodinámico coloidal propuesto Leontaritis (Leontaritis, 1987) representa un modelo matemático que se basa en el principio anterior con el fin de predecir la precipitación de asfaltenos. Sin embargo, sugiere el uso de la teoria de solución de polímero para representar el potencial químico de las resinas en la fase líquida, como se muestra en la Ecuación 27.

$$
\mu_{\text {resina }}^{\text {Fase liquida }}=\mu_{\text {resina }}^{\text {ref }}+R T\left(\ln \phi_{\text {resin }}+1-\frac{V_{\text {rvima }}}{V_{L}}+\frac{V_{\text {revima }}}{R T}\left(\delta_{\mathrm{L}}-\delta_{\text {resina }}\right)^{2}\right)(27)
$$

En la Ecuación 27, $\mu_{\text {resina }}^{\text {ref }}$ es el potencial químico de las resinas en forma pura a una $\mathrm{P}$ y $\mathrm{T}, \phi_{\text {resin }}$ es la fracción de volumen de las resinas en la fase liquida, $V_{\text {resina }}$ es el volumen molar de resinas, $V_{L}$ es el volumen molar promedio de la fase líquida remanente, $\delta_{\text {resina }}$ es el parámetro de solubilidad de resina, y $\delta_{L}$ es el parámetro de solubilidad medio de la fase liquida remanente.

\subsubsection{Modelo de solución polimérica}

En varios trabajos sobre la precipitación de asfaltenos, se ha sugerido que la teoría de solución polimérica puede ser utilizada para modelar la fase liquida en equilibrio con una fase de asfaltenos. La siguiente descripción se basa esencialmente en la de Hirschberg (Hirschberg et al., 1988). La Ecuación 28, expresa la fracción de volumen máximo de asfaltenos solubles en la fase liquida (aceite):

$$
\left(\varphi_{\mathrm{a}}\right)_{\max }=\exp \left(\frac{\mathrm{V}_{\mathrm{a}}}{\mathrm{V}_{\mathrm{L}}}\left[1-\frac{\mathrm{V}_{\mathrm{L}}}{\mathrm{V}_{\mathrm{a}}}-\frac{\mathrm{V}_{\mathrm{L}}}{\mathrm{RT}}\left(\delta_{\mathrm{a}}-\delta_{\mathrm{L}}\right)^{2}\right]\right)
$$

Donde, $V_{a}$, es el volumen molar del asfalteno, $V_{L}$, el Promedio de volumen molar del liquido, $\delta_{a}$ y $\delta_{L}$, son los parámetros de solubilidad de la fase asfalteno y liquida respectivamente. $V_{L}$ se halla utilizando una ecuación cúbica de estado, como se describe en la obra de Hirschberg (Hirschberg et al, 1988) usando la ecuación de Peng-Robinson modificada. El volumen molar de los asfaltenos se supone que es $\mathrm{Va}=4,000 \mathrm{~cm} 3 / \mathrm{mol}$. El parámetro de solubilidad lo definimos en la Ecuación 29;

$$
\delta^{2}=\frac{\Delta \mathrm{U}^{\mathrm{V}}}{\mathrm{V}}(29)
$$

Donde, $\Delta U^{V}$, es el cambio de energía en la vaporización isotérmica de un mol de líquido para un estado ideal de los gases. $V$, es el volumen molar para la fase líquida.

\subsubsection{Modelo de solución regular}

Este modelo propuesto (Chung, 1992) describe como un modelo predictivo generalizado que se basa en el principio termodinámico de equilibrio de fases sólido-líquido, se ha desarrollado para la precipitación de sólidos orgánicos. El modelo tiene en cuenta los efectos de la temperatura, composición y el coeficiente de actividad en 82 
la solubilidad de la cera y asfaltenos en soluciones orgánicas. E1 K-valor del equilibrio sólido-líquido se expresa como una función del calor de fusión, la temperatura de punto de fusión, parámetro de solubilidad y el volumen molar de cada componente en la solución. Todos estos parámetros se han correlacionado con el peso molecular. Así, el modelo se puede aplicar a los sistemas de aceite crudo.

\subsubsection{Modelo de solidos o de ecuación de estado}

Han propuesto un concepto de modelo (Ngheim et al, 1993) en el que se representan las fases gaseosa y líquida utilizando una ecuación cúbica de estado, mientras que la fase de asfaltenoses considerada como un sólido puro. La fugacidad $\left(f_{a}\right)$ de los asfaltenos se calcula a partir de la Ecuación 30 :

$$
\ln \left(f_{\mathrm{s}}\right)=\ln \left(\mathrm{f}_{\mathrm{s}}{ }^{*}\right)+\frac{\mathrm{Vs}^{*}\left(\mathrm{p} \cdot \mathrm{p}^{*}\right)}{\mathrm{R}^{*} \mathrm{~T}}(30)
$$

Donde, $P^{*}$ es la presión de referencia, $f_{s}^{*}$, es la fugacidad del sólido a $P^{*}$ y $V_{s}$, Volumen molar del sólido. Es necesario un algoritmo de cálculos flash multifásico para utilizar el modelo de asfaltenos mediante este modelo. Es claro resaltar que actualmente es uno de los modelos que tienen mayor precisión a la hora de realizar predicciones para precipitación de asfaltenos, para lograr esto requiere algunos datos experimentales para ajustar el modelo. El procedimiento realizado en esta investigación requiere las siguientes etapas para ser desarrollado:

- Caracterización del fluido

- Ajuste de coeficientes de interacción binaria

- Predicción del comportamiento de la precipitación

\subsubsection{Modelo de PC-SAFT}

Ese tipo de modelo ha encontrado hasta ahora un uso limitado en la industria del petróleo. La ecuación de PC-SAFT puede ser un buen candidato para cerrar la brecha entre los modelos de mecánica estadistica y los modelos clásicos de ingenieria de petróleo dominada por las ecuaciones de estado cúbicas. PC-SAFT es un modelo que ha sido desarrollado con base en el trabajo de Chapman (Chapman et al, 1990).

\subsubsection{Modelo de micelización}

Se ha trabajado un modelo (Victorov et al, 1996) de formación de micelas para la precipitación de asfaltenos a partir de aceites crudos. De acuerdo con este modelo, los asfaltenos se auto-asocian para formar un núcleo micelar esférico. Las moléculas de resina son adsorbidas sobre la superfície del núcleo micelar de asfaltenos formando una capa alrededor de ella. La precipitación es el resultado de minimizar la energía libre de Gibbs, cuando los asfaltenos en una fase infinitamente diluida se trasfieren a una fase de asfaltenos puro con una carcasa de resina. Para utilizar el concepto de este modelo, es necesario contar con modelos de fugacidad de asfaltenos, resinas y micelas resina-asfaltenos en solución, y un modelo para el cambio en la energía libre de Gibbs, como resultado de la formación de micelas. Debido a su complejidad, el modelo tiene un potencial limitado como modelo predictivo.

\subsection{Modelamiento de daño de formación por depositación de asfaltenos}

El daño más común encontrado cerca a la cara del pozo se debe a la depositación de asfaltenos, el cual puede ocurrir a mayores distancias dentro del yacimiento y en función de las caídas de presión. El modelo físico - mecánico presentado por Leontaritis (Leontaritis., 1996) predice de manera acertada el daño a la formación y la productividad debido a la depositación de asfaltenos.

El modelo se basa en el hecho de que es el crudo subsaturado el que exhibe procesos de precipitación y depositación de asfaltenos. Pese a esta limitante en esta investigación se ha llevado a cabo un proceso en el cual se puede trabajar inclusive a presiones por debajo de la presión de saturación, esto se logra gracias al modelo desarrollado anteriormente de precipitación de asfaltenos con ecuación de estado o también llamado modelo de sólidos. El fenómeno de floculación puede ocurrir cuando se produce a altas tasas de flujo. La presión en fondo de pozo $\left(\mathrm{P}_{\mathrm{w}}\right)$ es menor que la presión de inicio de floculación de asfaltenos $\left(\mathrm{P}_{\mathrm{AF}}\right)$, si el fluido no flocula ni 
deposita asfaltenos se considera un estado estable, por lo tanto el perfil de presión no cambia. Asumiendo que la presión del yacimiento $\left(\mathrm{P}_{\mathrm{q}}\right)$ no varía en un intervalo de tiempo estudiado, el caudal $(q)$ puede permanecer constante. Asi entonces, $P_{0}$ y $P_{A F}$ a $r_{0}$ y $r_{A F}$ respectivamente, permanecerán constantes si el caudal permanece constante, pero $P_{\mathrm{w}}$ disminuye continuamente para que el caudal q permanezca constante.

El modelo relaciona el área inicial al flujo con el área taponada por la depositación de asfaltenos (Ecuación 31 y 32), para determinar el grado de daño por depositación (DOD), Ecuación 33 :

$$
\begin{aligned}
& A_{\text {inicial }}=2 \pi \text { rh } \phi_{\text {inicial }}(31) \\
& \Delta A_{t\left(r_{1}, j\right)}=\Delta m_{t\left(r_{j}\right)} V_{A\left(r_{\mathrm{r}, j}\right)} \alpha_{A} \beta(32)
\end{aligned}
$$

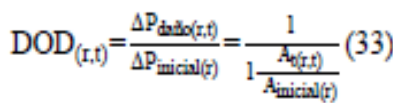

Donde $r$ es el radio, $h$ es el diámetro del poro, $\phi_{\text {inicial }}$ es la porosidad, el parámetro $\beta$ es un factor empirico que me representa la eficiencia de taponamiento, $\alpha_{A}$ es la superficie de las partículas retenidas, $V_{A}$ es el volumen molar del asfalteno y se determina mediante ecuación de estado y $\Delta m_{t(r, j)}$ es el número total de moles de asfalteno que han sido atrapadas a una distancia $\mathrm{r}$ y un tiempo $t$, el cual depende de la fracción de asfaltneno atrapada en la formación (ftrap) que se determina mediante:

$$
\mathrm{f}_{\text {trap }}=\left[\mathrm{d}_{A P_{\max }}\left(\psi-B \ln \left(\mathrm{d}_{\mathrm{AP} \max }\right)-1\right)\right]-\left[\mathrm{d}_{\mathrm{AP}, \mathrm{cr}}\left(\psi-\mathrm{B} \ln \mathrm{n}^{-2}\left(\mathrm{~d}_{\mathrm{AP}, \mathrm{cr}}\right)-1\right)\right](34)
$$

La Ecuación 34, se evalúa entre el diámetro de partícula crítico y el diámetro de partícula máximo. Cuando se determina el DOD, éste es utilizado entonces para hallar el perfil de presión a través del tiempo, la variación de la porosidad, permeabilidad y la progresión del daño usando las ecuaciones 35 a 38 .

$$
\begin{gathered}
\Delta \mathrm{P}_{\text {dã̃o(r,t) }}=\mathrm{DOD}_{(\mathrm{r}, \mathrm{t})} * \Delta \mathrm{P}_{\text {inicial }(\mathrm{r})} \\
\phi_{(\mathrm{r}, \mathrm{j})}=\frac{\phi_{(\mathrm{r}, \mathrm{j}-\mathrm{l})}}{\mathrm{DOD}(\mathrm{r}, \mathrm{j})}(36) \\
\mathrm{k}_{(\mathrm{r}, \mathrm{j})}=\frac{\mathrm{k}_{(\mathrm{r}, \mathrm{j}-\mathrm{l})}}{\mathrm{DOD}(\mathrm{r}, \mathrm{j})}(37) \\
\mathrm{S}_{(\mathrm{t})}=\Delta \mathrm{P}_{\mathrm{s}(\mathrm{t})} \frac{7.08 \mathrm{k}_{\text {imizis } \mathrm{h}}}{q \mu}(38)
\end{gathered}
$$

\subsection{Selección de modelos a trabajar y creación de un software computacional}

Se diseñó y desarrollo un software llamado "SMAP" (Software para el Modelamiento de Asfaltenos y Parafinas) que modela de una manera eficiente el comportamiento de sólidos como asfaltenos y parafinas, recopilando los modelos más robustos, con mayor efíciencia y aplicabilidad que hay en la actualidad.

Para el modelamiento de precipitación de asfaltenos se desarrollaron dos modelos, el primer modelo de solución polimérica se utiliza por su simplicidad a la hora de realizar cálculos ya que permite desarrollar cálculos mediante solo una composición del fluido en el yacimiento, el segundo modelo utilizado es el modelo de solidos por su exactitud, mayor cobertura, mejores ajustes y porque es el único modelo que permite el cambio composicional del fluido con el que trabajamos. Este modelo requiere mayor entrada de datos y ajustes experimentales. 
Para el modelamiento de la precipitación de parafinas se utilizaron el modelo de solución sólida y el modelo de fase multisólida, logrando con el primer modelo obtener cálculos de WAP (Punto de aparición de ceras), propiedades de fases liquida y sólida, además permite graficar el WEP (envolvente de precipitación de ceras), mientras que con el segundo modelo solo hallamos el WAP para crudos más pesados.

El modelo de daño de formación por depositación de asfaltenos, es una combinación de los trabajos realizados por Long Nghiem et al (1993) y Leontritis et al, (1996) el primero al plantear un modelo de precipitación "modelo de sólidos" ya mostrado anteriormente y el segundo autor al plantear un modelo físicomecánico que requiere alimentar datos de un modelo de precipitación de sólidos y que representa el daño en la formación con respecto al tiempo.

\section{Resultados}

Se tomaron diferentes muestras de aceite encontradas en la literatura y se modelo la precipitación el software SMAP, los resultados obtenidos se compararon con los datos experimentales y los obtenidos por otro software.

\subsection{Parafinas}

\subsubsection{Modelo de solución sólida}

Se realizó la prueba con un aceite tomado del campo escuela Colorado de la Universidad Industrial de Santander, se compararon los resultados de SMAP con los de los otros programas y se obtuvieron los resultados observados en la Tabla 1.

Tabla l. Resultados de los diferentes programas para determinación de WAP y la comparación con el SMAP.

\begin{tabular}{|c|c|c|c|c|}
\hline Presión (psi) & WAP experimental & Software & WAP ( $\left.{ }^{\circ} \mathrm{F}\right)$ & Error \% \\
\hline \multirow{4}{*}{814} & \multirow{4}{*}{130} & Was Calculator & 149.67 & 15 \\
\hline & & WimProp & 159 & 22 \\
\hline & & Ecopetrol & 86.28 & 33 \\
\hline & & SMAP & 113.5 & 13 \\
\hline \multirow{4}{*}{700} & \multirow{4}{*}{105} & Was Calculator & 149.3 & 42 \\
\hline & & WimProp & 158 & 50 \\
\hline & & Ecopetrol & -- & $\ldots$ \\
\hline & & SMAP & 111 & 5.7 \\
\hline \multirow{4}{*}{80} & \multirow{4}{*}{90} & Was Calculator & 147.58 & 63.9 \\
\hline & & WimProp & 158 & 75 \\
\hline & & Ecopetrol & --- & -- \\
\hline & & SMAP & 114.5 & 27.2 \\
\hline \multirow{4}{*}{14.7} & \multirow{4}{*}{90} & Wax Calculator & 147.58 & 63.9 \\
\hline & & WimProp & 158 & 75 \\
\hline & & Ecopetrol & 103.68 & 15.2 \\
\hline & & SMAP & 113.5 & 26.1 \\
\hline
\end{tabular}

\subsubsection{Modelo de multisólido}

La prueba se realizó con un fluido tomado del trabajo de Lira Galena (Lira Galena et al., 1996) y se determinó con el software SMAP el punto de aparición de parafinas (WAP) y se comparó con los reportados en la literatura como se observa en la Tabla 2, teniendo en cuenta que para éste crudo el WAP experimental fue de $554.76 \mathrm{R}$

Tabla 2. Puntos de aparición de parafinas (WAP) para el aceite 3.

\begin{tabular}{l|l|l}
\hline Software utilizado & WAP $(\mathrm{R})$ & \% error \\
\hline CMG & 601.2 & 8.37 \\
\hline WAX-SYM & 558.67 & 0.70 \\
\hline WAP-SIM & 554.923 & 0.029 \\
\hline SMAP & 556.7 & 0.34 \\
\hline
\end{tabular}




\subsection{Asfaltenos}

\subsubsection{Modelo de sólido o de ecuación de estado}

Para la validación del modelo de precipitación de asfaltenos, el software se corrió para varias composiciones a las cuales se le había determinado el asfalteno precipitado a diferentes presiones. Estos datos están reportados en la literatura (Burke et al., 1990) y se obtuvieron los resultados observados en la Tabla 3:

Tabla 3. Precipitación de asfaltenos a dos presiones y a una temperatura de $212^{\circ} \mathrm{F}$

\begin{tabular}{l|c|c|c|c|c}
\hline \multirow{2}{*}{ Presiones } & \multicolumn{2}{|c|}{ Porcentaje de Asfalteno precipitado (\%wt) } & \multicolumn{2}{c}{$\%$ error } \\
\cline { 2 - 6 } & Experimental & SMAP & CMG & SMAP & CMG \\
\hline 1014.7 & 0.403 & 0.417 & 0.12220 & 3.47 & 69.67 \\
\hline 4014.7 & 0.402 & 0.420 & 0.40208 & 4.47 & 0.019 \\
\hline
\end{tabular}

Además de esto el software genera graficas de precipitación de asfalteno, la cual también se comparó por las generadas por el CMG, Figura 1 .
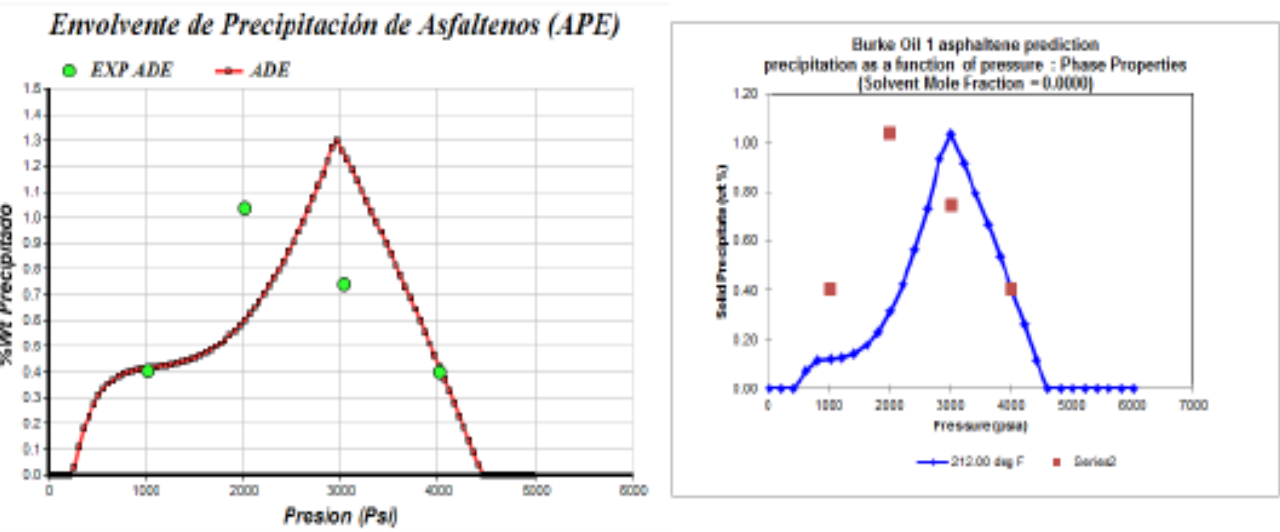

Figura 1. Datos experimental vs Modelo de Sólidos del SMAP para el aceite 1 a una temperatura de $212^{\circ} \mathrm{F}$

\subsection{Depositación de asfaltenos}

SMAP genera gráficas para analizar el comportamiento de la presión, porosidad, permeabilidad y factor de daño partiendo del modelo de precipitación de asfaltenos, datos PVT, de la formación y del pozo. Figura 2.

\section{Conclusiones}

Se seleccionaron los modelos termodinámicos de precipitación de parafinas; Modelo de solución sólida y Modelo de fase multisólida. En cuanto a lo relacionado con precipitación de asfaltenos se ha realizado una exhaustiva clasificación de modelos existentes en la actualidad, como conclusión hemos clasificado 6 tipos, de los cuales seleccionamos los dos mejores modelos; Modelo de sólidos y Modelo de solución polimérica.

Para predecir asfaltenos el modelo que mejor responde respecto a datos experimentales es el modelo de sólidos, pero este modelo requiere de mayor información y algunos datos experimentales. Mientras que el modelo de solución polimérica es un modelo que permite un fácil trabajo y solo requiere la composición del fluido, cabe resaltar que no es muy preciso ni permite ajustes. 

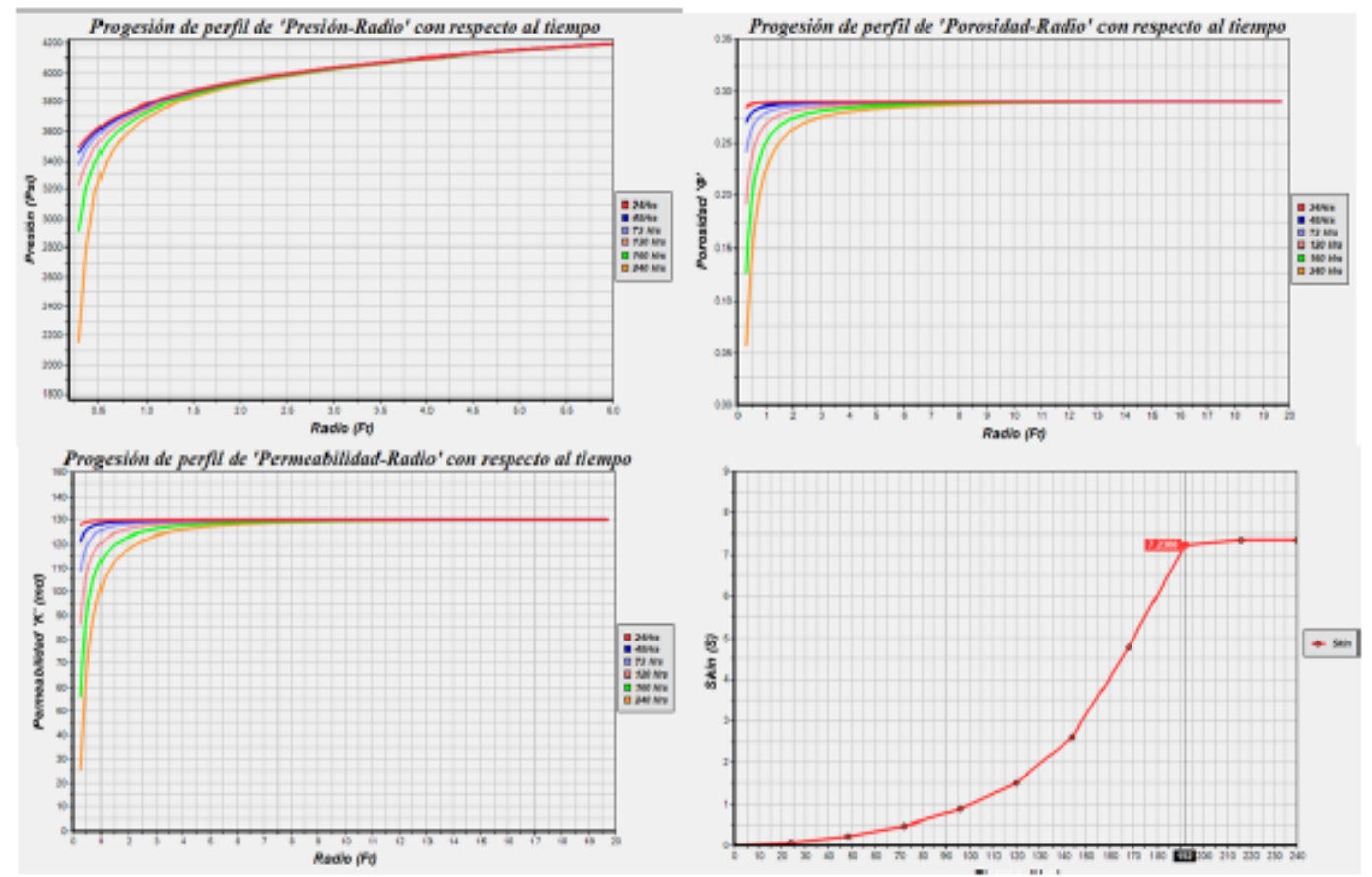

Figura 2. Gráfica que muestra la progresión de la presión, porosidad, permeabilidad y factor de daño skin a través del tiempo

La precipitación de parafinas cuenta con dos teorias y modelos muy aceptados hoy en dia, por ello ambos se desarrollaron en el software, mostrando muy buenos datos de predicción de temperatura de aparición de parafinas (WAP). E1 modelo que presenta adicionalmente la cantidad de solido precipitado a una presión y temperatura dada es el modelo de solución sólida.

Una vez concluido los modelos a trabajar, se desarrolló un software que tiene la capacidad de caracterizar una mezcla de hidrocarburos, realizar cálculos de equilibrio Líquido-Vapor, Realizar cálculos de equilibrio Líquido-Vapor-Sólido, Modelar la precipitación de asfaltenos, modelar la precipitación de parafinas, predecir el daño de formación por depositación de sólidos y construir Gráficas de envolventes de fases (P-T, APE, WED, ADE) y progresión de perfiles (Presión-Radio, Porosidad-Radio, Permeabilidad-Radio, Skin-Tiempo).

\section{Referencias Bibliográficas}

1. Burke, N. E., Hobbs, R. E., Kashon, S.F., 1990. Measurement and Modeling of Asphaltene Precipitation, Joumal of Petroleum Technology, 1440-1446p.

2. Chapman, W.G., Gubbins, K.E., Jackson, G., Radosz, M., 1990. New Reference Equation of State for Associating Liquids, Ind Eng. Chem. Res. 29, 1709-1721p.

3. Chung, T., 1992. Thermodynamic Modeling for Organic Solid Precipitation, paper SPE 24851 presented at the SPE 67 th Annual Technical Conference and Exhibition held in Washington, DC.

4. Hirschberg, A., De Jong, L. N. J., Schipper, B. A., Meyers, J. G., 1988. Influence of Temperature and Pressure on Asphaltene Flocculation, paper SPE 11202 presented at the SPE 57th Annual Technical Conference and Exhibition held in New Orleans, LA. 
5. Leontanitis, K. J., Mansoori, G. A., 1987. Asphaltene Flocculation During Oil Production and Processing: A Thermodynamic Colloidal Model, paper SPE 16258 presented at the SPE International Symposium on Oilfield Chemistry held in San, Antonio, TX, U. S.

6. Leontaritis, KJ., 1996. Asphaltene Near-wellbore Formation Damage Modeling. Society of Petroleum Engineers (SPE) 39446.

7. Lira-Galena, C., Firoozabadi, A., Prausnitz J.M., 1996. Thermodynamic Of Wax Precipitation in Petroleum Mixtures, AIChE J., vol. 42, 239-248p.

8. Nghiem, L. X. and Aziz, K. 1984. A Robust Iterative Method for Flash Calculations Using the Soave-RedlichKwong or the Peng Robinson Equiation of State. SPE 8285.

9. Nghiem, L. X, Hassam, M. S., Nutakki, R., George, A. E. D, 1993. Efficient Modeling of Asphaltene Precipitation", paper SPE 20530 presented at the SPE 68th Annual Technical Conference and Exhibition held in Houston, Texas.

10. Nichita, D.V., Goual L., Firoozabadi A., 2001. "Wax Precipitation in Gas Condensate Mixtures," SPE Prod. Facil., vol. 16,250-259p

11. Pedersen, K.S., Skovborg, P., Ronningsen, H.P., 1991. Wax Precipitation from North Sea Crude Oils 4. Thermodynamic Modeling, Energy and Fuels, vol. 5, 924-932p.

12. Ronningsen, H.P., Somme, B.F., Pedersen, K.S., 1997. An Improved Thermodynamic Model for Wax Precipitation: Experimental Foundation and Application. Paper presented at 8th Intemational Conference on Multiphase 97, Cannes, France.

13. Victorov, A.I, Firoozabadi, A., 1996. Thermodynamics of Asphaltene Deposition Using a Micellization Model, AIChE J. 42, 1753-1764p.

14. Won, K.W., 1986. Thermodynamics for Solid Solution-Liquid-Vapor Equilibria: Wax Phase Formation from Heavy Hydrocarbon Mixtures, Fluid Phase Equilib., vol. 30, 265-279p.

15. Won, KW., 1989. Thermodynamic Calculation of Cloud Point Temperatures and Wax Phase Compositions of Refined Hydrocarbon Mixtures, Fluid Phase Equilibria 53, 377-396p. 